\title{
Cytokines in innate host defense in the lung
}

\author{
Robert M. Strieter, John A. Belperio, and Michael P. Keane \\ Department of Medicine, Division of Pulmonary and Critical Medicine, University of California-Los Angeles \\ School of Medicine, Los Angeles, California, USA \\ Address correspondence to: Robert M. Strieter, Department of Medicine, Division of Pulmonary and Critical Care Medicine, \\ UCLA School of Medicine, 900 Veteran Avenue, 14-154 Warren Hall, Box 711922, Los Angeles, California 90024-1922, USA. \\ Phone: (310) 794-1999; Fax: (310) 794-1998; E-mail: rstrieter@mednet.ucla.edu.
}

J. Clin. Invest. 109:699-705 (2002). DOI:10.1172/JCI200215277.

Historically the lung has been perceived as an organ primarily involved in gas exchange. However, due to its unique relationship with the environment, the lung must defend itself from infection by numerous inhaled micro-organisms. Various innate defenses protect the lung from infection, including the cough reflex, mucociliary clearance (see Knowles and Boucher, this Perspective series, ref. 1), and antimicrobial properties of the mucosal surface (see Ganz, this series, ref. 2; and McCormack and Whitsett, this series, ref. 3). In addition, an extensive alveolar-capillary membrane containing immune and nonimmune cells is exposed to microbial challenges. Consequently, pulmonary tissues generate a brisk innate host response to both inhaled and hematogenous pathogens in order to clear the offending micro-organism and preserve gas exchange.

The successful execution of the innate defense response in the lung is critical to the eventual transition and development of the adaptive immunity. Once containment of the micro-organism has occurred, the response resolves, leading to repair or tissue remodeling. Loss of these later responses leaves the lung susceptible to tissue injury caused by excessive local or distant innate responses. In conditions such as acute respiratory distress syndrome, overexuberant tissue inflammation in response to micro-organisms may lead to irreversible lung injury and mortality.

While a variety of factors - arachidonic acid metabolites, coagulation factors, complement, acute-phase proteins, and antimicrobial peptides among them - are involved in the innate response, cytokines constitute the largest and most pleiotropic group of such mediators and will be the focus of this Perspective. The initiation, maintenance, and resolution of pulmonary innate responses depend upon cellular communication via cytokines. Along with other soluble factors, as well as adhesion molecules, the cytokines contribute to the recognition of pathogens, the recruitment of neutrophils and mononuclear cells, and the removal of the invading micro-organism.

Cytokine signaling occurs through receptor-ligand interactions on specific immune or nonimmune target cell populations. These populations differ not only in their complement of cytokine receptors, but also in their capacities to elaborate and secrete specific cytokines in response to particular stimuli. Interactions among various cell populations have led to the concept of cytokine networking, in which one population of cells may respond directly to specific exogenous or endogenous stimuli, leading to the elaboration of a particular cytokine that exerts distinct effects upon another population of cells. The targets respond by producing cytokines, which may serve as feedback signals to the initiating cell, or, alternatively, by releasing signaling molecules that affect yet another array of target cells. Inflammatory effector cells, such as neutrophils and monocytes, may be locally recruited and activated in response to specific chemotactic signals, resulting in further amplification of a cytokine cascade by nonimmune resident cells.

\section{Micro-organism recognition \\ by the innate immune system}

Evolution has provided the mammalian host with two major forms of host defense, innate and adaptive immune responses (4). The innate defense is the gatekeeper and sentry for immediate host defense against invading micro-organisms, whereas the adaptive immune response is naive, is delayed in development, and must be taught through somatic generation of a diverse repertoire of receptors prior to the full development of an appropriate immune response to invading microbes (4). This difference in behavior suggests that innate responses must be based on the recognition of micro-organism-associated molecular patterns. The adaptive immune response, in contrast, depends on two classes of specialized lymphocytes, $\mathrm{T}$ and $\mathrm{B}$ cells, whose specific receptors are somatically generated in response to antigen presentation by professional antigen-presenting cells - dendritic cells, macrophages, and other B cells. This process causes antigen-dependent clonal expansion of $\mathrm{T}$ and $\mathrm{B}$ cells, resulting in long-term humoral and cell-mediated immune memory. However, acquired immunity does not occur immediately in response to a novel antigen or pathogen, and a delay in response could have a devastating effect on host survival. Therefore, the innate 
and acquired immune responses are coordinated, with innate immunity representing the initial and instructive process in host defense.

Micro-organisms express molecular patterns that are unique and readily distinguished from host. These include viral double-stranded RNA; unmethylated CPG dinucleotides common in bacterial DNA but underrepresented in vertebrate DNA; mannans of yeast; glycolipids of mycobacteria; lipoproteins of bacteria and parasites; lipoteichoic acids of Gram-positive bacteria; and LPS of Gram-negative bacteria. The host has evolved specific pattern recognition receptors to detect these pathogen-associated molecules. These receptors can be divided into secreted, endocytic, and signaling classes of receptors (4), the last of which induce the expression of a variety of cytokines that subsequently amplify innate defense.

\section{Toll-like receptors mediate pattern recognition signaling}

Cytokines that stimulate the innate immune response are not constitutively expressed but must be called into play by specific signals that alert the host to invading micro-organisms. Mammalian Toll-like receptors (TLRs), important signaling receptors in innate host defense, are similar in structure and function to the Drosophila Toll protein (4). The cytoplasmic domain of this transmembrane protein is similar to that of the mammalian IL-1 receptor, suggesting that both Toll and mammalian TLRs share similar signal-transduction pathways via a MyD88-dependent pathway that ultimately involves the NF- $\kappa \mathrm{B}$ family of transcriptional factors. NF- $\kappa B$ serves as a master switch, transactivating various cytokines that are involved in the innate and transition to adaptive immunity (5).

Medzhitov and colleagues were the first to characterize a human TLR, TLR4 (6). The constitutively active mutant of TLR4, when transfected into human cell lines, activates NF- $\kappa \mathrm{B}$ and stimulates the expression of the proinflammatory cytokines IL-1, -6, and -8. In addition, TLR4 signal transduction and NF- $\kappa B$ transactivation induces expression of IL-12 p40, as well as CD80 and CD86, costimulatory molecules that link innate and adaptive immune responses by activating antigen-specific responses by naive T cells. TLR4 recognizes Gramnegative bacteria via LPS signal transduction and is present on macrophages, dendritic cells, and B cells. In LPS-resistant $\mathrm{C} 3 \mathrm{H} / \mathrm{HeJ}$ and $\mathrm{C} 57 \mathrm{BL} / 10 \mathrm{ScCr}$ mice, defective LPS signaling is caused by mutations in the Tlr 4 gene (7). However, LPS recognition and triggering of the innate host response is more complex than direct interaction with TLR4. LPS first interacts with a serum protein, the LPS-binding protein, which transfers LPS to the membrane-anchored protein CD14. CD14 lacks a transmembrane and cytoplasmic domain for signaling coupling, but it appears to use TLR4 as a coreceptor (8). Another protein, MD-2, associates constitutively with TLR4 and confers enhanced responsiveness to LPS. Hence, host recognition of LPS is dictated by a complex of at least three components, CD14, TLR4, and MD-2 (4). In contrast, TLR2 recognizes components of Grampositive bacteria such as peptidoglycan (9). Interesting- ly, it has recently been shown that renal epithelial cells constitutively express both TLR2 and TLR4. This expression is upregulated by both IFN- $\gamma$ and TNF during renal inflammation (10), suggesting that nonimmune cells can play an important role in innate host defense.

\section{Recognition cytokines and the initial response to pulmonary pathogens}

While TLRs mediate pathogen recognition by the innate immune system in response to unique molecular patterns presented by the micro-organism, it is increasingly clear that cytokines are necessary for the full development of the innate host defense and the transition to adaptive immunity. Early-response cytokines are activated after TLR signal-coupling and, in turn, amplify and engage additional cells in the response to invading micro-organisms. Two of the most important early-response cytokines in innate immunity are IL-1 and TNF.

\section{The IL-1 family of cytokines}

The IL-1 family of cytokines consists of two agonists, IL- $1 \alpha$ and IL- $1 \beta$, and one antagonist, IL-1 receptor antagonist (IL-1Ra). The IL-1 agonists are encoded by two distinct genes that produce protein isoforms, IL- $1 \alpha$ and IL- $1 \beta$. IL- $1 \alpha$ is predominantly membrane-associated, while IL- $1 \beta$ is secreted. IL- $1 \alpha$ and IL- $1 \beta$ are produced by a variety of cells, and both bind to the type I IL-1 receptor on target cells, eliciting similar biologic functions (11).

IL-1 binding to the IL-1 type I receptor and the IL-1 receptor-associated protein recruits an adapter molecule, MyD88, which in turn recruits the IL-1 receptor-associated kinase (IRAK). IRAK recruits the TNF receptor-associated factor 6 (TRAF6), an adapter molecule, which complexes with the NF- $\mathrm{KB}$-inducing kinase (NIK). NIK activates the IKB kinase complex (IKK), which phosphorylates $I \kappa B \alpha$, leading to ubiquitination and degradation of $\mathrm{I} \kappa \mathrm{B}$. NF- $\kappa \mathrm{B}$, released from its association with $\mathrm{I} \kappa \mathrm{B} \alpha$, translocates to the nucleus and transactivates numerous target genes, encoding cyclooxygenase, adhesion molecules, cytokines, NO synthase, acute-phase proteins, and various cytokines and chemokines (12). While IL-1 signaling can occur through two other pathways (the p38/mitogen-activated protein [MAP] kinase and the c-Jun N-terminal kinase [JNK] pathways), their effects often synergize with those of NF- $\kappa \mathrm{B}$ activation. Interestingly, the signal coupling of IL-1 and the IL-1 type I receptor is identical to that of LPS on TLR4 (4). These two divergent ligandreceptor pairs ultimately signal through the same cytoplasmic pathway, leading to NF- $\mathrm{BB}$ activation, nuclear translocation, and transactivation of several genes critical to the amplification of the innate immunity. This apparent redundancy suggests that $\mathrm{NF}-\kappa \mathrm{B}$ activation is critical for the innate response and for the initiation of the adaptive immune response. Thus, endogenous ligands, such as IL-1, can further amplify the LPS response by activating NF- $\kappa \mathrm{B}$, even in cells that do not express the CD14/TLR4/MD-2 complex. Since IL-1 receptors are present on essentially all immune and nonimmune cells, IL-1 can bind to the IL-1 type I receptor and engage most cells in the innate immune response. 
IL-1 also binds to an IL-1 type II or "decoy" receptor, which does not signal. Binding of IL-1 to the IL-1 type II receptor may be a mechanism to sequester IL-1, blocking its interaction with the IL-1 type I receptor (11). The IL-1 type II receptor is cleaved by metalloproteases on the cell surface, releasing a soluble form of the IL-1 type II receptor, which retains its ability to bind IL- $1 \beta$ and inhibit IL-1 signaling. The IL-1 pathway is modulated at yet another level by expression of IL-1Ra, a naturally occurring inhibitor of IL-1 binding. IL-1Ra consists of three isoforms derived from alternatively spliced mRNAs produced from a single gene encoding secreted and intracellular forms. Thus, a dynamic balance between IL-1 agonists and IL-1Ra influences IL-1-dependent inflammation. A variety of immune and nonimmune cells express IL-1Ra, which competitively antagonizes IL-1 $\alpha$ and IL- $1 \beta$, attenuating IL-1 activity in vitro and in vivo (11). Intracellular isoforms of IL-1Ra are released after cellular injury or apoptosis, inhibiting IL-1 signaling and, perhaps, contributing to the resolution of inflammatory responses after injury. Indeed, the intratracheal injection of LPS, IL-1, or TNF causes intra-alveolar infiltration by neutrophils, followed later by mononuclear cell infiltrates. LPS also induces TNF and IL-1 expression in the lung, further amplifying the inflammatory response. Conversely, IL-1Ra reduces inflammatory responses to LPS in the lungs, and the production of IL-1Ra by mononuclear phagocytes and other cells in the lung may influence innate host response.

Surprisingly, analysis of the inflammatory responses in $I L-1 \beta$ gene-targeted mice has not supported a requirement for IL- $1 \beta$ signaling in innate defense of the lung, since generation of IL-1 $\alpha$, IL- 6 , and TNF and sensitivity to killing by LPS are similar in $I L-1 \beta^{-/-}$ and wild-type mice (13). Thus, IL-1 $\beta$ signaling contributes to but is not essential for a systemic inflammatory response. While IL-1 initiates and modifies inflammatory cascades, redundancy, as well as alternative signaling pathways, allows for the induction and maintenance of innate inflammatory responses even in the absence of IL- $1 \beta$.

\section{TNF}

TNF is primarily a mononuclear phagocyte-derived cytokine that has pleiotropic effects on innate host responses to micro-organisms. TNF signals through a unique family of receptors. The TNF homotrimer binds to two different cell surface receptors, p55 and $\mathrm{p} 75$. The $\mathrm{p} 55$ receptor, like a related protein, the Fas receptor, contains a 60 -amino acid domain known as the "death domain," which is essential for signal transduction of an apoptotic signal (14). As with IL-1 and LPS signaling, TNF signaling occurs through NIK and MAP/extracellular signal-regulated kinase $1 / 2$ (MEK1/2), which activate NF- $\mathrm{KB}$, in turn enhancing transcription of genes mediating innate immune responses. Thus, three distinct receptors, TLRs, IL-1R, and TNF receptors, induce NF- $\kappa B$ nuclear translocation and transcription of genes critical to the perpetuation of innate immune responses (Figure 1).
A diverse array of extracellular and intracellular bacteria and fungi trigger the expression of TNF. TNF is increased in patients with pneumonia and is expressed early in animal models of pulmonary infection caused by Streptococcus pneumoniae, Klebsiella pneumoniae, Pseudomonas aeruginosa, Legionella pneumophila, Cryptococcus neoformans, Aspergillus fumigatus, and Pneumocystis carinii (15-17). Depletion of TNF impairs clearance of micro-organisms from the lung $(15,17)$. While TNF does not directly mediate the migration of neutrophils, it regulates neutrophilendothelial cell adhesion molecules and chemotactic factors, the CXC chemokines, which recruit inflammatory cells to the site of infection. Expression of TNF in the lung using a recombinant adenoviral vector can stimulate infiltration by neutrophils and can markedly improve bacterial clearance and survival after challenge with intrapulmonary $K$. pneumoniae. The importance of TNF in host defense can be seen in the increased incidence of tuberculosis reported in a large cohort of patients who had received anti-TNF therapy (infliximab) (18).

\section{Recruitment cytokines}

The extravasation of neutrophils, eosinophils, basophils, and mononuclear cells is the salient feature of the innate response to micro-organisms in the lung.

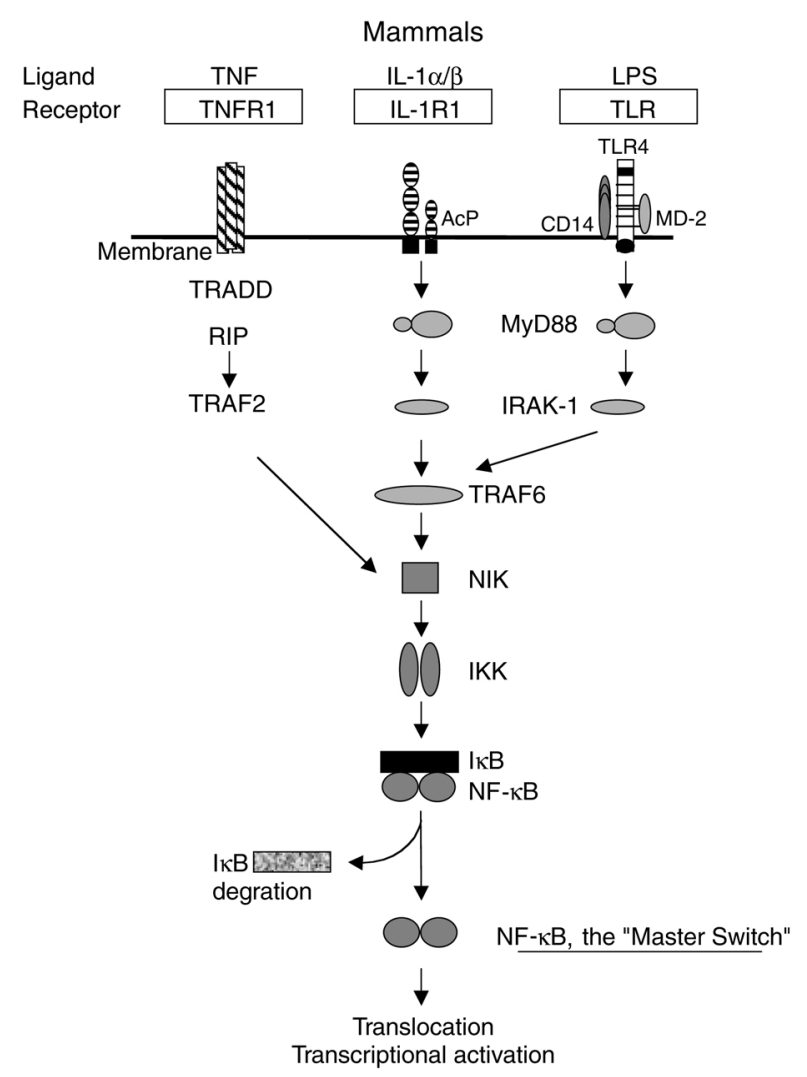

Figure 1

The triumvirate of TLR, IL-1, and TNF signal transduction, activation, and translocation of NF-KB as the "master switch" for transactivation of a number of cytokine genes involved in mediating innate host defense. TNFR1, TNF receptor 1; TRADD, TNFR1-associated death domain; RIP, receptor-interacting protein. 
Extravasating leukocytes eradicate the invading microorganisms and contribute to the inflammatory response. The initiation and maintenance of leukocyte recruitment during lung inflammation require communication among infiltrating leukocytes, the endothelium, and resident stromal and respiratory epithelial cells. The production of chemotactic molecules, such as chemokines, plays a critical role in this process of recruitment and maintenance of inflammatory cells following infection.

The human CXC, CC, C, and CX3C chemokines, as they are called in a relatively new classification scheme (19), are four closely related polypeptide families that are potent chemotactic factors for neutrophils, eosinophils, basophils, monocytes, mast cells, dendritic cells, NK cells, and T and B lymphocytes. CXC chemokines can be further divided into two groups on the basis of a structure/function domain consisting of the presence or absence of three amino acids (Glu-LeuArg; the "ELR" motif) preceding the first cysteine amino acid residue. The ELR ${ }^{+}$CXC chemokines are chemoattractants for neutrophils and act as potent angiogenic factors. In contrast, the $\mathrm{ELR}^{-} \mathrm{CXC}$ chemokines are chemoattractants for mononuclear leukocytes, and ELR- IFN-inducible CXC chemokines potently inhibit angiogenesis (19).

Chemokines are produced by an array of cells including monocytes, alveolar macrophages, neutrophils, platelets, eosinophils, mast cells, T and B lymphocytes, NK cells, epithelial cells, fibroblasts, smooth muscle cells, mesothelial cells, and endothelial cells. These cells can produce chemokines in response to a variety of factors that trigger the innate immune response, including pattern recognition ligands, IL-1, TNF, C5a, leukotriene B4 (LTB4), and IFNs. Their production by both immune and nonimmune cells supports the view that chemokines play a pivotal role in orchestrating both the innate immune response and the transition to adaptive immunity.

\section{Posttranslational processing of chemokines}

Leukocyte chemotactic factors vary in their longevity at sites of inflammation. For example, while C5a is rapidly inactivated to its Des-Arg form in the presence of serum/plasma peptidases, IL-8 (CXCL8) retains its activity in the presence of serum. Thrombin and plasmin (or neutrophil granule lysates and purified proteinase-3) convert IL-8 (CXCL8) from the 77-amino acid to the 72-amino acid form, enhancing its biological activity. The 72-amino acid form of IL-8 (CXCL8) has higher affinity for neutrophils than does the 77-amino acid form, and it is two- to threefold more potent in inducing cytochalasin B-treated neutrophil degranulation. Activity of ENA-78 (CXCL5) is also influenced by proteolysis of $\mathrm{NH}_{2}$-terminal peptides.

$\mathrm{NH}_{2}$-terminal processing of $\mathrm{CC}$ chemokines also influences their ability to recruit mononuclear cells. CD26/dipeptidyl peptidase IV, a lymphocyte membrane-associated peptidase, selectively cleaves peptides with proline or alanine at the second position and cleaves dipeptides at the $\mathrm{NH}_{2}$-terminus. While $\mathrm{NH}_{2}$-terminal truncation of the CXC chemokine granulocyte chemotactic protein-2 (CXCL6) by CD26 does not alter neutrophil chemotactic activity, $\mathrm{NH}_{2}$-terminal truncation by CD26 markedly impairs the chemotactic activity of RANTES (CCL5), eotaxin (CCL11), and macrophage-derived chemokine (CCL22). $\mathrm{NH}_{2}$-terminal truncated RANTES (CCL5) binds with normal affinity to CCR5 but is impaired in its activation of CCR 1 and CCR3. Interestingly, $\mathrm{NH}_{2}$-terminal processing by $\mathrm{CD} 26$ of LD78 $\beta$, an isoform of macrophage inflammatory peptide- $1 \alpha$ (MIP- $1 \alpha$; CCL3), increases its chemotactic activity, an effect mediated by the chemokine receptors CCR1 and CCR5 (20). Thus, extracellular processing of leukocyte chemoattractants alters chemokine receptor selectivity, modifies the ability of these molecules to recruit leukocytes, and influences the development of subsequent adaptive immunity.

\section{Chemokine receptors}

Chemokine receptors belong to the largest known family of cell surface receptors, the $G$ protein-coupled receptors, which also mediate stimuli from hormones, peptides, glycopeptides, and chemokines. The temporal-spatial regulation of chemokines and chemokine receptors further influences inflammatory responses. Thus, neutrophils expressing CXCR 1 and CXCR 2 arrive early in response to ELR ${ }^{+}$CXC chemokines, which are generated in response to micro-organism recognition. Monocytes and other mononuclear cells expressing CCR2, CCR1, and CCR5 arrive later in response to monocyte chemoattractant protein-1 (MCP-1; CCL2), MIP-1 $\alpha$ (CCL3), and RANTES (CCL5). These elicited leukocytes play a critical role in the eradication of the micro-organism. In addition, specific chemokine ligand-receptor pairs can recruit immature or mature dendritic cells. These cells then present antigens to $T$ and $B$ cells in the secondary lymphoid tissues, activating the latter cells and thus bridging the innate and adaptive immune responses to micro-organisms (21). Chemokines and their receptors help effect the shift to adaptive immunity by several other means, as well - acting as costimulatory molecules for $\mathrm{T}$ cell activation, behaving as chemoattractants for naive and activated subpopulations of lymphocytes and antigen-presenting cells, establishing functional microenvironments in secondary lymphoid tissue for adaptive immunity, and inducing polarization of Th 1 and Th 2 cytokine-mediated immune responses (22).

\section{The role of chemokines in host defense}

ELR $^{+}$CXC chemokines contribute to neutrophil sequestration in the lungs of patients with pneumonia. IL-8 (CXCL8) has been found in the bronchoalveolar lavage of patients with community-acquired pneumonia and nosocomial pneumonia following trauma (23, 24). $\mathrm{ELR}^{+} \mathrm{CXC}$ chemokines, including growth-related gene (GRO- $\alpha$; CXCL1; KC) and GRO- $\beta / \gamma(\mathrm{CXCL} 2 / 3$; MIP-2), were identified in lung preparations taken from rabbits with Escherichia coli pneumonia and from mice with K. pneumoniae, P. aeruginosa, Nocardia asteroides, and A. fumigatus pneumonia $(17,25-27)$.

In the mouse, $\mathrm{ELR}^{+} \mathrm{CXC}$ ligands, several of which are expressed in murine models of pneumonia, bind to the 
receptor CXCR2. Standiford and associates used specific neutralizing antibodies to CXCR2, demonstrating that blocking of CXCR2 reduces neutrophil infiltration in response to $P$. aeruginosa (28), $N$. asteroides (27), and $A$. fumigatus (29) pneumonias. CXCR2 blockade also reduces clearance of the micro-organisms and increases mortality, demonstrating the importance of $\mathrm{ELR}^{+} \mathrm{CXC}$ chemokine/CXCR2 biology in the innate host defense. Tsai and associates (26) demonstrated that enhanced expression of murine GRO- $\alpha$ (KC; CXCL1) in Clara cells improved host survival during $K$. pneumoniae pneumonia. Thus, the compartmentalized overexpression of this $\mathrm{ELR}^{+} \mathrm{CXC}$ chemokine could be used to treat pneumonia without relying on antibiotics - a potentially important approach given the increasing prevalence of multi-drugresistant micro-organisms in clinical practice.

The CC chemokines RANTES (CCL5), MIP-1 $\alpha$ (CCL3), MIP-1 $\beta$ (CCL4), and MCP-1 (CCL2) have been implicated in innate host defense in animal models of influenza A virus (30), Paramyxovirus pneumonia virus, A. fumigatus, and C. neoformans pneumonias. The host response to influenza A virus is characterized by an influx of mononuclear cells into the lungs and increased expression of CC chemokine ligands. Dawson and colleagues (30) used a genetic approach to identify a role of $\mathrm{CC}$ chemokines in innate response to this virus. They noted increased mortality related to severe pneumonitis following influenza A viral pneumonia in $C C R 5^{-/}$mice but found that $C C R 2^{-/-}$mice were protected from the severe pneumonitis due to defective macrophage recruitment. The delay in macrophage accumulation in $C C R 2^{-/}$mice correlated with increased pulmonary viral titers (30). These studies show that the CC chemokines and chemokine receptors play distinct roles during influenza A viral infection and demonstrate the importance of macrophage recruitment during the innate response to this pathogen.

The effect of CC chemokines in the recruitment of mononuclear cells during the innate host defense of the lung is not limited to viral infections. C. neoformans is acquired via the respiratory tract and is a significant cause of fatal mycosis in immunocompromised patients. Both the innate and the adaptive immune response are necessary to clear the microbe from the lung and to prevent dissemination to the meninges. Huffnagle and associates (31) have found that MCP-1 (CCL2) and MIP-1 $\alpha$ (CCL3) play important roles in the eradication of $C$. neoformans from the lung and prevent meningitis. MIP-1 $\alpha$ (CCL3) is also needed for the efficient clearance of $K$. pneumoniae pulmonary infection (32). Mice deficient in this chemokine suffer significantly greater mortality than wild-type mice following challenge with $K$. pneumoniae. This mutation does not affect leukocyte recruitment, but it significantly impairs phagocytic activity of alveolar macrophages (32).

\section{Th1 and Th2 cytokines in the clearance of pulmonary pathogens}

The discovery by Mosmann and colleagues that Th cell subsets could be classified on the basis of the cytokines they express (33) provides further insight into the transition from the innate to adaptive immune responses to bacterial infection. The Th 1 and Th 2 cytokine patterns in mice were originally identified from a panel of Th cell clones. The Th1 pattern includes IFN- $\gamma$ and IL-2, and the Th2 pattern includes IL-4, IL-5, and IL-10. These cytokines are produced not only by Th cells, but also by other $\mathrm{T}$ cells and other cells unrelated to leukocytes. The lists of known Th1 (IFN- $\gamma$, IL-2, TNF, IL-12, and IL-18) and Th2 (IL-4, IL-5, IL-6, IL-10, and IL-13) cytokines have expanded subsequently.

Th1 cytokines are involved in activation of macrophages and neutrophils, augmentation of professional phagocyte-dependent functions, and direction of cell-mediated immunity. In contrast, Th2 cytokines inhibit a variety of innate host response functions, including various macrophage and neutrophil activities; generate humoral immune responses and antibody production; and promote eosinophil colony stimulatory activity. The balance of Th1 and Th2 cytokines influences innate host responses and is key to the transition to cellular and humoral adaptive immunity and the removal of the inciting antigen. IFN- $\gamma$ promotes the differentiation of Th 1 cells and suppresses Th2 cytokine responses, while IL-4 and IL-10 shift the immune response toward a Th2-like cytokine pattern, promoting Th2 cell differentiation and suppressing Th1 cell activity. In vitro studies show that IL-10 and IL-4 inhibit the production of IL-2, whereas IFN- $\gamma$ can augment its expression.

\section{The pivotal role of IFN- $\gamma$ in innate defense}

IL-18 and IL-12 are Th1 cytokines that induce the expression of IFN- $\gamma$ from NK and T cells, cells critically involved in innate and adaptive immune responses, respectively. IFN- $\gamma$ is a pivotal Th1 cytokine with multiple effects on $T$ cell recruitment and phagocyte-mediated clearance of pathogens. First, this cytokine potentiates the respiratory burst and activity of professional phagocytes and enhances generation of nitric oxide by these cells. In addition, it promotes the expression of MHC class I and II antigens on a variety of cells, priming them to present class-restricted antigen (34). IFN- $\gamma$ also regulates the expression of Fc receptors on professional phagocytic cells, and it induces the expression of ICAM-1 as well as the IFN-inducible CXC chemokines and CC chemokines in a variety of cells, thus amplifying the recruitment of additional leukocytes to a site of innate host response. The recruitment of Th1 and other mononuclear cells expressing the putative CXC chemokine receptor CXCR3 leads to further amplification of the local expression of IFN- $\gamma$. In T cells, IFN- $\gamma$ induces the expression of the costimulatory molecules CD80 and CD86, priming the cells for antigen-dependent activation. It also inhibits the expression of CD40 ligand on activated Th1 and Th2 cells and suppresses a number of ELR ${ }^{+}$CXC chemokines from a variety of cells $(19,35)$. The suppression of ELR ${ }^{+}$CXC chemokines by IFN- $\gamma$ and the induction of ELR ${ }^{-}$IFN-inducible CXC and $\mathrm{CC}$ chemokines may be important mechanisms for switching from a predominantly neutrophilic to a mononuclear cell infiltrate. IFN- $\gamma$ also promotes proliferation of antigen-stimulated $\mathrm{T}$ cells and generation of 
CD8 cytotoxic $\mathrm{T}$ cells that are directly involved in the adaptive immune response. Finally, it promotes IgG1 switching to IgG2 in B cells. These properties place IFN- $\gamma$ at a central position to orchestrate a number of biological events related to the full development of the innate host response, the transition from innate to adaptive immunity, and the polarization of the adaptive immune response to promote cell-mediated immunity.

An example of the importance of IFN- $\gamma$ in mediating host defense in the lung is seen following pulmonary infection with Mycobacterium tuberculosis (TB). IFN $\gamma^{\prime-}$ animals are unable to contain an otherwise sublethal pulmonary inoculation of TB $(36,37)$. Conversely, exogenous IFN- $\gamma$ can be helpful in treating this disease. Condos and associates (38) administered aerosolized IFN- $\gamma$ to patients with multi-drug-resistant TB. IFN- $\gamma$ treatment was well tolerated, increased or stabilized body weight, decreased the size of cavitary lesions, and reduced time to negative culture (38). With increasing antibiotic resistance in TB and other bacteria, IFN- $\gamma$ may be useful as an adjunct to therapy for lung infections that fail to respond to conventional antimicrobial therapy. Indeed, it has recently been shown in a murine model that transient pulmonary overexpression of IFN- $\gamma$ (and, to a lesser extent, IL-12) aids in the clearance of $L$. pneumophila in vivo and enhances killing of extracellular organisms ex vivo (39).

\section{IL-10 as a modulator of innate responses}

IL-10, a Th2 cytokine that inhibits innate and adaptive immune activities, blocks the expression of proinflammatory cytokines, including IFN- $\gamma$, IL-1, TNF, IL-12, and $\mathrm{CXC}$ and CC chemokines. It suppresses MHC class II and costimulatory molecules CD80 and CD86 on macrophages, and it inhibits the generation of reactive oxygen and nitrogen intermediates from macrophages and neutrophils. Nevertheless, this cytokine increases the expression of the $\mathrm{Fc}$, $\mathrm{fMLP}$, and PAF receptors, as well as the chemokine receptor CCR5. Taken together, these data suggest that IL-10 impairs the direct killing of micro-organisms but augments mononuclear phagocyte and neutrophil recruitment to a site of microbial invasion and thus enhances phagocytosis of micro-organisms.

While exogenous administration of IL-10 protects the lung from injury in response to LPS or immune-complex deposition (40), IL-10 can be detrimental to the host under conditions of micro-organism invasion. For example, Greenberger and associates (41) demonstrated that IL-10 mRNA and protein are markedly increased in the lung during the pathogenesis of K. pneumoniae pneumonia. Depletion of IL-10 augments intrapulmonary expression of TNF, the ELR ${ }^{+}$CXC chemokine, MIP-2 (CXCL2/3), and the CC chemokine MIP-1 $\alpha$ (CCL3), thus increasing pulmonary clearance of $K$. pneumoniae and improving host survival. Steinhauser et al. observed the inhibitory effects of IL-10 during sepsis and pneumonia in an unusual murine model (42). They found that mortality was greater in mice subjected first to cecal ligation and puncture and then challenged with intrapulmonary $P$. aeruginosa, as compared with mice challenged with intrapulmonary $P$. aeruginos $a$ in the absence of cecal ligation and puncture. The increased mortality was attributed to increased bacteremia and reduced lung bacterial clearance and was associated with increased levels of IL-10. Depletion of endogenous IL-10 in the mice that underwent cecal ligation and puncture prior to challenge with $P$. aeruginosa reduced bacteremia, increased bacterial clearance, and improved survival.

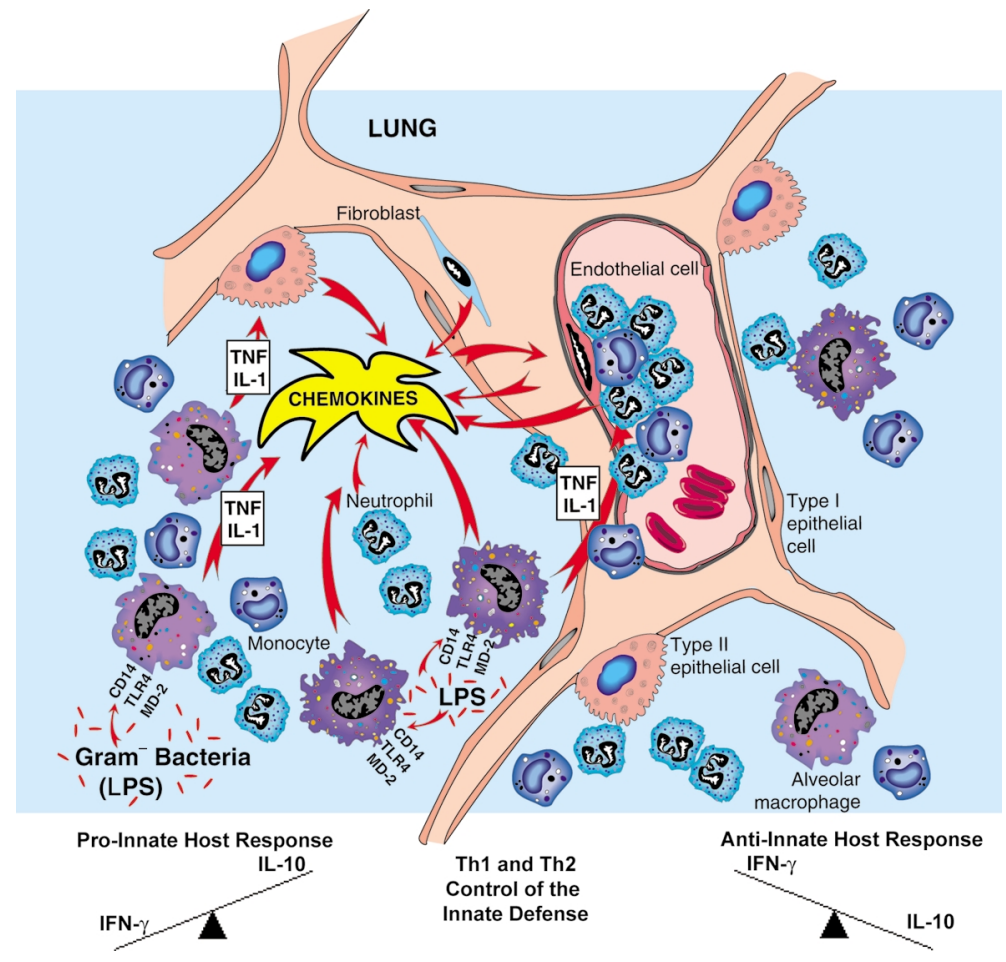

Figure 2

The importance of the events related to recognition, recruitment, and removal of invading micro-organisms during the development of the innate host defense. 


\section{Conclusions}

The lung has a unique relationship with the environment and, through evolution, has developed strategies to defend the host from invasion by numerous microorganisms. The full development of an appropriate innate host defense in the lung requires the recognition of microbial molecular patterns by the TLRs and other signaling pathways that contribute to their effects. These receptors enhance expression of IL- $1 \beta$ and TNF, in turn amplifying cellular responses to the pathogen. IL-1 $\beta$ and TNF engage immune and nonimmune cells, which are recruited by chemokines and cytokines and contribute to eradicating an infection (Figure 2). A balance of Th1 and Th2 cytokines modulates the intensity of the response, and anti-inflammatory cytokines suppress inflammation during recovery. Cytokines and chemokines serve to orchestrate a transition from innate to adaptive immunity.

As we encounter increasing numbers of micro-organisms with resistance to antimicrobials, we will need to gain further knowledge of innate defense systems to design novel strategies for preventing or treating pulmonary infection. Such therapies must be achieved without causing exuberant inflammation that ultimately injures the lung and increases mortality of the host.

\section{Acknowledgments}

Due to space constraints, a number of important references could not be included in this article. Interested readers can find a suggested reading list at www.jci.org/cgi/content/full/109/6/699/dc1. This work was supported, in part, by NIH grants PO1 HL-67665 (to M.P. Keane and R.M. Strieter), HL-03906 (to M.P. Keane), CA-87879 and HL-60289 (to R.M. Strieter), and HL-04493 (to J.A. Belperio). M.P. Keane is the holder of a Dalsemer Scholar Research Award from the American Lung Association.

1. Knowles, M.R., and Boucher, R.C. 2002. Mucus clearance as a primary innate defense mechanism for mammalian airways. J. Clin. Invest. 109:571-577. DOI:10.1172/JCI200215217.

2. Ganz, T. 2002. Antimicrobial polypeptides in host defense of the respiratory tract. J. Clin. Invest. 109:693-697. DOI:10.1172/JCI200215218.

3. McCormack, F.X., and Whitsett, J.A. 2002. The pulmonary collectins SP-A and SP-D orchestrate innate immunity in the lung. J. Clin. Invest. 109:707-712. DOI:10.1172/JCI200215293.

4. Medzhitov, R., and Janeway, C., Jr. 2000. Innate immunity. N. Engl. J. Med. 343:338-344.

5. Ghosh, S., May, M.J., and Kopp, E.B. 1998. NF-kappa B and Rel proteins: evolutionarily conserved mediators of immune responses. Annu. Rev. Immunol. 16:225-260.

6. Medzhitov, R., Preston-Hurlburt, P., and Janeway, C.A., Jr. 1997. A human homologue of the Drosophila Toll protein signals activation of adaptive immunity. Nature. 388:394-397.

7. Hoshino, K., et al. 1999. Cutting edge. Toll-like receptor 4 (TLR4)-deficient mice are hyporesponsive to lipopolysaccharide: evidence for TLR4 as the Lps gene product. J. Immunol. 162:3749-3752.

8. Beutler, B., and Poltorak, A. 2000. Positional cloning of Lps, and the general role of toll-like receptors in the innate immune response. Eur. Cytokine Netw. 11:143-152.

9. Yoshimura, A., et al. 1999. Cutting edge: recognition of Gram-positive bacterial cell wall components by the innate immune system occurs via Toll-like receptor 2. J. Immunol. 163:1-5.

10. Wolfs, T.G., et al. 2002. In vivo expression of Toll-Like receptor 2 and 4 by renal epithelial cells: IFN-gamma and TNF-alpha mediated up-regulation during inflammation. J. Immunol. 168:1286-1293.

11. Dinarello, C.A. 1998. Interleukin-1 beta, interleukin-18, and the interleukin-1 beta converting enzyme. Ann. NY Acad. Sci. 856:1-11.

12. Murphy, J.E., Robert, C., and Kupper, T.S. 2000. Interleukin-1 and cuta- neous inflammation: a crucial link between innate and acquired immunity. J. Invest. Dermatol. 114:602-608.

13. Fantuzzi, G., et al. 1996. Effect of endotoxin in IL-1 beta-deficient mice. J. Immunol. 157:291-296.

14. Bazzoni, F., and Beutler, B. 1996. The tumor necrosis factor ligand and receptor families. N. Engl. J. Med. 334:1717-1725.

15. Laichalk, L.L., et al. 1996. Tumor necrosis factor mediates lung antibacterial host defense in murine Klebsiella pneumonia. Infect. Immun. 64:5211-5218.

16. Brieland, J.K., et al. 1995. In vivo regulation of replicative Legionella pneumophila lung infection by endogenous tumor necrosis factor alpha and nitric oxide. Infect. Immun. 63:3253-3258.

17. Mehrad, B., Strieter, R.M., and Standiford, T.J. 1999. Role of TNF-alpha in pulmonary host defense in murine invasive aspergillosis. J. Immunol. 162:1633-1640.

18. Keane, J., et al. 2001. Tuberculosis associated with infliximab, a tumor necrosis factor alpha-neutralizing agent. N. Engl. J. Med. 345:1098-1104.

19. Zlotnik, A., and Yoshie, O. 2000. Chemokines: a new classification system and their role in immunity. Immunity. 12:121-127.

20. Proost, P., et al. 2000. Cleavage by CD26/dipeptidyl peptidase IV converts the chemokine LD78beta into a most efficient monocyte attractant and CCR1 agonist. Blood. 96:1674-1680.

21. Sozzani, S., Allavena, P., Vecchi, A., and Mantovani, A. 2000. Chemokines and dendritic cell traffic. J. Clin. Immunol. 20:151-160.

22. Gu, L., et al. 2000. Control of TH2 polarization by the chemokine monocyte chemoattractant protein-1. Nature. 404:407-411.

23. Boutten, A., et al. 1996. Compartmentalized IL-8 and elastase release within the human lung in unilateral pneumonia. Am. J. Respir. Crit. Care Med. 153:336-342.

24. Rodriguez, J.L., et al. 1992. Local production of interleukin-8 is associated with nosocomial pneumonia. J. Trauma. 33:74-81.

25. Mehrad, B., and Standiford, T.J. 1999. Role of cytokines in pulmonary antimicrobial host defense. Immunol. Res. 20:15-27.

26. Tsai, W.C., et al. 1998. Lung-specific transgenic expression of KC enhances resistance to Klebsiella pneumoniae in mice. J. Immunol. 161:2435-2440.

27. Moore, T.A., et al. 2000. Bacterial clearance and survival are dependent on CXC chemokine receptor-2 ligands in a murine model of pulmonary Nocardia asteroides infection. J. Immunol. 164:908-915.

28. Tsai, W.C., et al. 2000. CXC chemokine receptor CXCR2 is essential for protective innate host response in murine Pseudomonas aeruginosa pneumonia. Infect. Immun. 68:4289-4296.

29. Mehrad, B., et al. 1999. CXC chemokine receptor-2 ligands are necessary components of neutrophil-mediated host defense in invasive pulmonary aspergillosis. J. Immunol. 163:6086-6094.

30. Dawson, T.C., Beck, M.A., Kuziel, W.A., Henderson, F., and Maeda, N. 2000. Contrasting effects of CCR5 and CCR2 deficiency in the pulmonary inflammatory response to influenza A virus. Am. J. Pathol. 156:1951-1959.

31. Huffnagle, G.B., et al. 1997. Macrophage inflammatory protein-1alpha (MIP-1alpha) is required for the efferent phase of pulmonary cell-mediated immunity to a Cryptococcus neoformans infection. J. Immunol. 159:318-327.

32. Lindell, D.M., Standiford, T.J., Mancuso, P., Leshen, Z.J., and Huffnagle, G.B. 2001. Macrophage inflammatory protein 1alpha/CCL3 is required for clearance of an acute Klebsiella pneumoniae pulmonary infection. Infect. Immun. 69:6364-6369.

33. Mosmann, T.R., Cherwinski, H., Bond, M.W., Giedlin, M.A., and Coffman, R.L. 1986. Two types of murine helper T cell clone. I. Definition according to profiles of lymphokine activities and secreted proteins. J. Immunol. 136:2348-2357.

34. Driggers, P.H., et al. 1990. An interferon gamma-regulated protein that binds the interferon-inducible enhancer element of major histocompatibility complex class I genes. Proc. Natl. Acad. Sci. USA. 87:3743-3747. 35. Rollins, B.J. 1997. Chemokines. Blood. 90:909-928.

36. Cooper, A.M., et al. 1993. Disseminated tuberculosis in interferon gamma gene-disrupted mice. J. Exp. Med. 178:2243-2247.

37. Flynn, J.L., et al. 1993. An essential role for interferon gamma in resistance to Mycobacterium tuberculosis infection. J. Exp. Med. 178:2249-2254

38. Condos, R., Rom, W.N., and Schluger, N.W. 1997. Treatment of multidrug-resistant pulmonary tuberculosis with interferon-gamma via aerosol. Lancet. 349:1513-1515.

39. Deng, J.C., Tateda, K., Zeng, X., and Standiford, T.J. 2001. Transient transgenic expression of gamma interferon promotes Legionella pneumophila clearance in immunocompetent hosts. Infect. Immun. 69:6382-6390.

40. Standiford, T.J., Strieter, R.M., Lukacs, N.W., and Kunkel, S.L. 1995. Neutralization of IL-10 increases lethality in endotoxemia. Cooperative effects of macrophage inflammatory protein-2 and tumor necrosis factor. J. Immunol. 155:2222-2229.

41. Greenberger, M.J., et al. 1995. Neutralization of IL-10 increases survival in a murine model of Klebsiella pneumonia. J. Immunol. 155:722-729.

42. Steinhauser, M.L., et al. 1999. IL-10 is a major mediator of sepsis-induced impairment in lung antibacterial host defense. J. Immunol. 162:392-399. 